\title{
A cost-effective way to monitor for ophthalmic complications during prone surgery
}

T Roberts ${ }^{1}$, D Burckett-St-Laurent ${ }^{2}$, October 2016

1. Specialist Registrar, Welsh School of Anaesthesia

2. Consultant, Royal Gwent Hospital, Newport, UK

Email: tomroberts@doctors.org.uk

\section{Aim}

1. To identify a cost-effective and safe way to monitor patients' eyes during prone surgery.

2. To demonstrate that introducing new equipment into clinical use can be achieved without great difficulty.

\section{Background}

Vision loss following spinal surgery is a rare but life-changing complication and has been documented since the early 20th century (1). There are several mechanisms of post-operative vision loss (POVL) associated with surgery in the prone position.

Overall, estimates for the incidence of POVL range from 0.028 to $0.2 \%$ (2) making it an extremely rare complication, however the devastating nature of the effects on the patient means that prevention of POVL should be a high priority for this patient group.

We describe a novel and cost-effective way to monitor for ophthalmic complications during prone surgery.

Products such as the Dupaco Proneview ${ }^{\circledR}$ (3) allow visualisation of the eyes during surgery to guard against external ocular pressure and direct trauma to the eye. However, in some circumstances, particularly in cervical spine surgery, the patient is positioned with their head at the opposite end of the theatre to the anaesthetic machine. The head is often covered in drapes and it can be difficult to gain access to regularly assess the eyes. It was suggested that a more cost-effective way to allow clear, regular monitoring of the eyes could be developed. Dupaco also manufacture a face mask incorporating video monitoring, however the cost is prohibitively expensive for many centres.

\section{Method}

We identified a commercially available (non-medical) camera and video monitor which had the ability to focus as close as $5 \mathrm{~mm}$, operate in very low light and that could be easily attached to the Proneview ${ }^{\circledR}$ (or alternative) mask. The total cost of these items was £24. The camera itself was $£ 7$, a low enough cost to be considered a disposable item in case of contamination.

Permission to use the equipment was sought from the hospital EBME department (Electrical and Biomechanical Engineering). The equipment was exempt from formal testing as it was battery-powered and was CE marked. Two positions for the positioning of the camera were identified. The camera can either be placed directly underneath the eyes, facing upwards, or can be taped to the side of the Proneview ${ }^{\circledR}$ face mask, pointing at the mirror below. The equipment was tested using simulated conditions prior to being used on real patients.

Users were provided with comprehensive instructions for the setup and use of the device. Individual users could choose to either leave the screen on constantly, or to turn it on to look at the eyes at regular intervals.

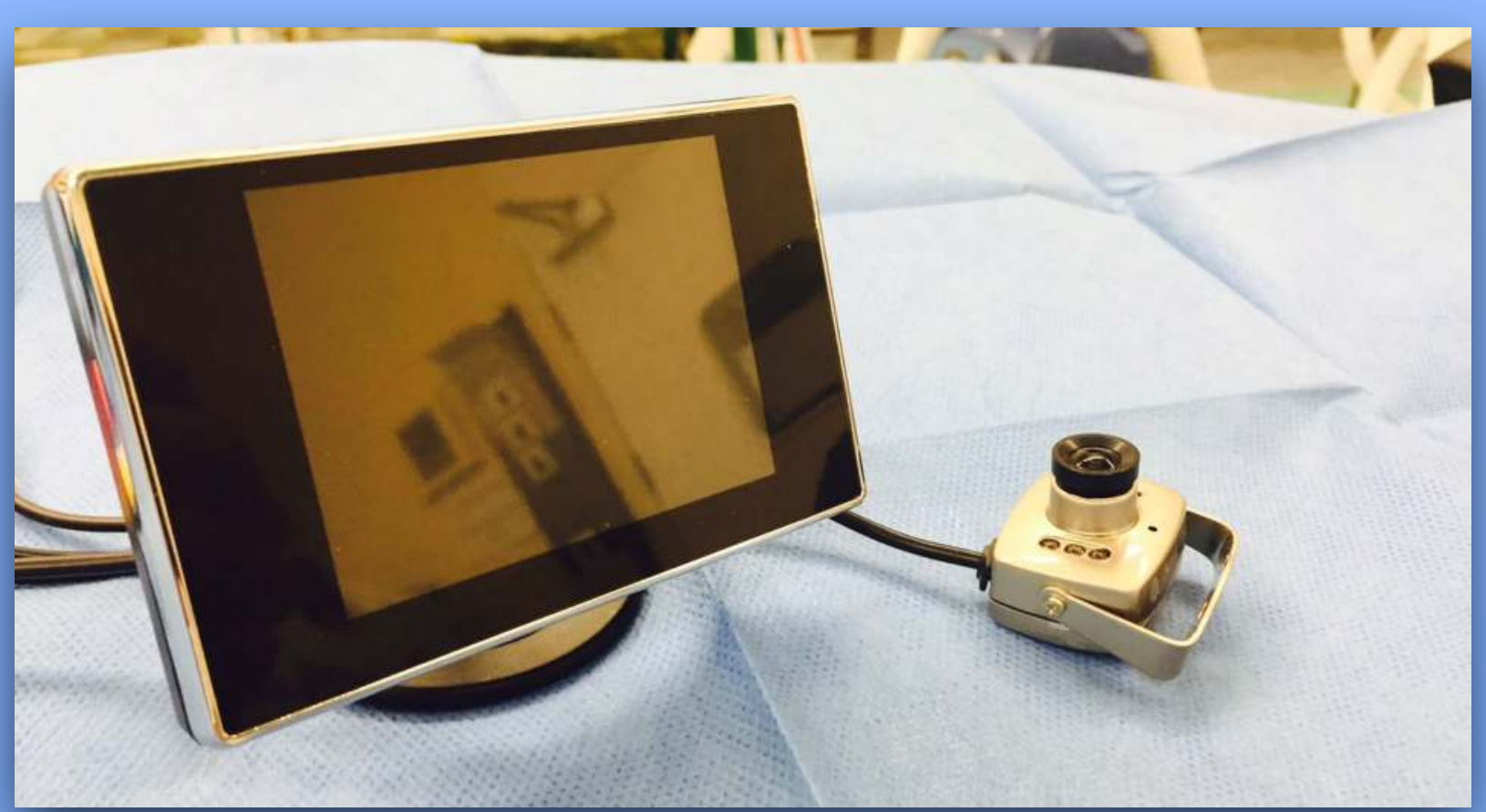

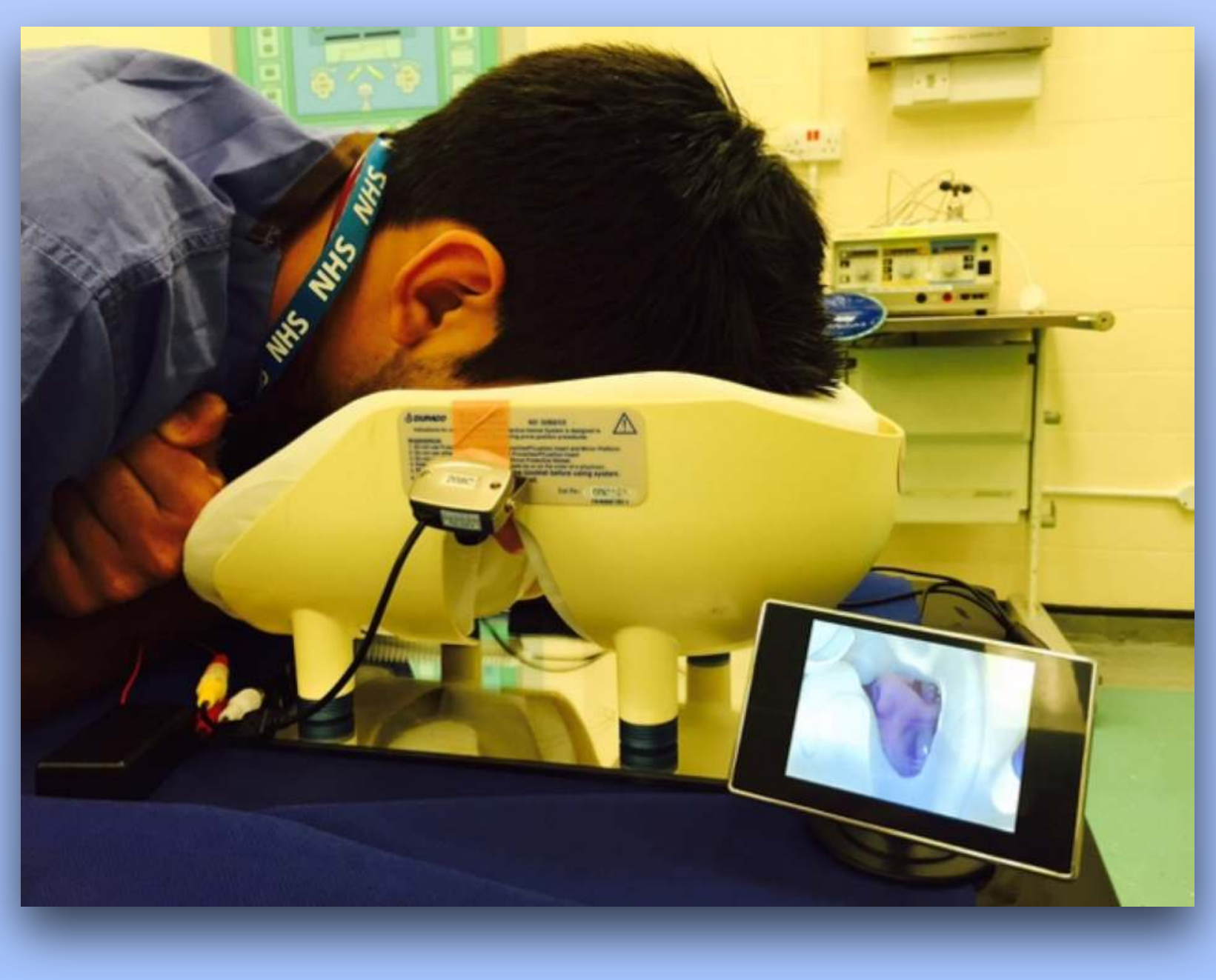

Evaluation and Feedback

Feedback was sought from a number of consultants and trainees in the local anaesthetic department. In summary:

- There is no benefit in using this equipment routinely for lumbar spinal surgery.

- In situations where the patient's head is positioned away from the machine, particularly for cervical spine surgery, the camera makes it easier to monitor the eyes.

- Anaesthetists were likely to check the eyes more regularly when using the camera than they would have done without it.

- Diathermy can cause interference when using the monitor. The interference is reduced by shielding the video cable within a $2 \mathrm{~m}$ length of breathing circuit tubing. In addition, the monitor can be switched off when diathermy is in use.

- Placing the camera directly below the eyes facing upwards is more likely to lead to contamination from blood, cleaning fluid etc.

\section{Conclusion}

For selected surgical cases where the patient is in the prone position, our equipment has been shown to provide an effective and low-cost method of monitoring for ophthalmic complications.

Whilst rare, ophthalmic complications can have a devastating impact on a patient's quality of life and further development of these types of products will contribute to patient safety initiatives.

Introducing new equipment can be straightforward and anaesthetists should not be put off by perceived bureaucratic hurdles in the pursuit of innovation.
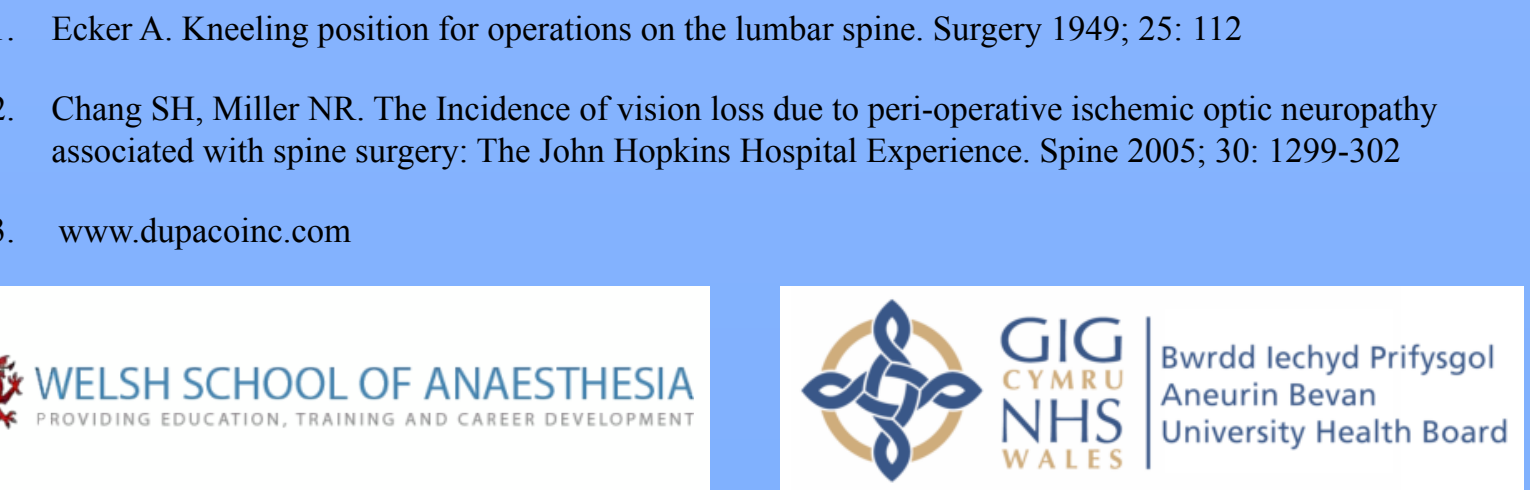